\title{
On Nonlinear Dirichlet-Neumann Algorithms for Jumping Nonlinearities
}

Heiko Berninger, Ralf Kornhuber, and Oliver Sander

FU Berlin, FB Mathematik und Informatik

(http://www.math.fu-berlin.de/rd/we-02/numerik/)

Summary. We consider a quasilinear elliptic transmission problem where the nonlinearity changes discontinuously across two subdomains. By a reformulation of the problem via Kirchhoff transformation we first obtain linear problems on the subdomains together with nonlinear transmission conditions and then a nonlinear SteklovPoincaré interface equation. We introduce a Dirichlet-Neumann iteration for this problem and prove convergence to a unique solution in one space dimension. Finally we present numerical results in two space dimensions suggesting that the algorithm can be applied successfully in more general cases.

\section{Introduction}

Let $\Omega \subset \mathbb{R}^{n}$ be a bounded Lipschitz domain divided into two non-overlapping subdomains $\Omega_{1}, \Omega_{2}$ with the interface $\Gamma=\bar{\Omega}_{1} \cap \bar{\Omega}_{2}$.

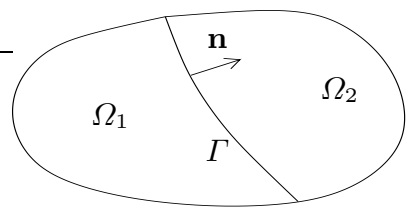

Fig. 1. Non-overlapping partition of the domain $\Omega$.

Given $f \in L^{2}(\Omega), k_{1}, k_{2} \in L^{\infty}(\mathbb{R})$ with $k_{i} \geq \alpha>0$ for $i=1,2$ we consider the following quasilinear elliptic transmission problem:

Find a function $p$ on $\Omega, p_{\mid \Omega_{i}}=p_{i} \in H^{1}\left(\Omega_{i}\right), i=1,2, p_{\mid \partial \Omega}=0$, such that

$$
\begin{aligned}
-\operatorname{div}\left(k_{i}\left(p_{i}\right) \nabla p_{i}\right) & =f & & \text { on } \Omega_{i}, i=1,2 \\
p_{1} & =p_{2} & & \text { on } \Gamma \\
k_{1}\left(p_{1}\right) \nabla p_{1} \cdot \mathbf{n} & =k_{2}\left(p_{2}\right) \nabla p_{2} \cdot \mathbf{n} & & \text { on } \Gamma .
\end{aligned}
$$


Observe that the nonlinearities $k_{i}$ need not be differentiable. Hence, Newton-type linearization suffers from a lack of smoothness. However, by standard Kirchhoff transformation [1], we can reformulate the two nonlinear pdes (1) as linear Poisson equations in each of the subdomains. Based on this observation, we introduce a nonlinear Dirichlet-Neumann algorithm for (1)-(3) which requires the solution of two linear problems in each iteration step but does not involve any further linearization. We present a convergence analysis including sufficient conditions for convergence in one space dimension. As a by-product, we obtain an existence and uniqueness result for (1)-(3). Numerical computations suggest that the algorithm can also be applied successfully to higher-dimensional problems. Related Robin methods will be treated in a forthcoming paper [3].

This paper is organized as follows. In Section 2 we apply the Kirchhoff transformation to (1)-(3) in order to derive an interface problem with linear problems on the subdomains and nonlinear transmission conditions. The transformed problem can be rewritten as a nonlinear Steklov-Poincaré interface equation. In analogy to the linear case, the nonlinear Dirichlet-Neumann algorithm can be regarded as a preconditioned Richardson iteration applied to the nonlinear Steklov-Poincaré equation. In Section 3, we present a convergence theorem in 1D generalizing related results in the linear case [5]. Finally, in Section 4, we illustrate the numerical properties of the nonlinear DirichletNeumann method in a nontrivial two-dimensional setting.

\section{An elliptic problem with jumping nonlinearities}

We introduce (for $i=1,2$ ) the spaces

$$
V_{i}:=\left\{v_{i} \in H^{1}\left(\Omega_{i}\right) \mid v_{i \mid \partial \Omega \cap \partial \Omega_{i}}=0\right\}, \quad V_{i}^{0}:=H_{0}^{1}\left(\Omega_{i}\right), \quad \Lambda:=H_{00}^{1 / 2}(\Gamma)
$$

and for $w_{i}, v_{i} \in V_{i}$ the forms

$$
a_{i}\left(w_{i}, v_{i}\right):=\left(\nabla w_{i}, \nabla v_{i}\right)_{\Omega_{i}}, \quad b_{i}\left(w_{i}, v_{i}\right):=\left(k\left(w_{i}\right) \nabla w_{i}, \nabla v_{i}\right)_{\Omega_{i}},
$$

where $(\cdot, \cdot)_{\Omega_{i}}$ stands for the $L^{2}$ inner product on $\Omega_{i}$. The norm in $H^{1}\left(\Omega_{i}\right)$ will be denoted by $\|\cdot\|_{1, \Omega_{i}}$, the norm in $\Lambda$ with $\|\cdot\|_{\Lambda}$. We point out that much of what we present in the first two sections are generalizations of the linear theory given in [5]. The notation here is used accordingly.

Let $R_{i}, i=1,2$, be any continuous extension operator from $\Lambda$ to $V_{i}$. Then the variational formulation of problem (1)-(3) reads as follows:

Find $p_{i} \in V_{i}, i=1,2$, such that

$$
\begin{array}{rlrl}
b_{i}\left(p_{i}, v_{i}\right) & =\left(f, v_{i}\right)_{\Omega_{i}} \quad \forall v_{i} \in V_{i}^{0}, \quad i=1,2 \\
p_{1 \mid \Gamma} & =p_{2 \mid \Gamma} \quad & \text { in } \Lambda & \\
b_{1}\left(p_{1}, R_{1} \mu\right)-\left(f, R_{1} \mu\right)_{\Omega_{1}} & =-b_{2}\left(p_{2}, R_{2} \mu\right)+\left(f, R_{2} \mu\right)_{\Omega_{2}} & \forall \mu \in \Lambda .
\end{array}
$$


We now introduce new variables $u_{i}, i=1,2$, by Kirchhoff transformation $\kappa_{i}$ (cf. [1]):

$$
u_{i}(x):=\kappa_{i}\left(p_{i}(x)\right)=\int_{0}^{p_{i}(x)} k_{i}(q) d q \text { a.e. in } \Omega_{i},
$$

which yields $k_{i}\left(p_{i}\right) \nabla p_{i}=\nabla u_{i}$. Further properties of $\kappa_{i}$ are listed in the following

Proposition 1. $\kappa_{i}: \mathbb{R} \rightarrow \mathbb{R}$ is a.e. differentiable with $\kappa_{i}^{\prime}=k_{i}$, strictly monotonically increasing and Lipschitz continuous with Lipschitz constant $\left\|k_{i}\right\|_{\infty}$. $\kappa_{i}^{-1}$ is Lipschitz continuous with Lipschitz constant $\left\|k_{i}^{-1}\right\|_{\infty}^{-1}$.

Furthermore there exist positive constants $c, C$ with

$$
\begin{gathered}
c\left\|p_{i}\right\|_{1, \Omega_{i}} \leq\left\|\kappa_{i}\left(p_{i}\right)\right\|_{1, \Omega_{i}} \leq C\left\|p_{i}\right\|_{1, \Omega_{i}} \text { and } \\
c\left\|p_{i \mid \Gamma}\right\|_{\Lambda} \leq\left\|\kappa_{i}\left(p_{i}\right)_{\mid \Gamma}\right\|_{\Lambda} \leq C\left\|p_{i \mid \Gamma}\right\|_{\Lambda} .
\end{gathered}
$$

Thus with this transformation problem (4)-(6) becomes: Find $u_{i} \in V_{i}, i=1,2$, such that

$$
\begin{aligned}
a_{i}\left(u_{i}, v_{i}\right) & =\left(f, v_{i}\right)_{\Omega_{i}} \quad \forall v_{i} \in V_{i}^{0}, \quad i=1,2 \\
\kappa_{1}^{-1}\left(u_{1 \mid \Gamma}\right) & =\kappa_{2}^{-1}\left(u_{2 \mid \Gamma}\right) \quad \text { in } \Lambda \\
a_{1}\left(u_{1}, R_{1} \mu\right)-\left(f, R_{1} \mu\right)_{\Omega_{1}} & =-a_{2}\left(u_{2}, R_{2} \mu\right)+\left(f, R_{2} \mu\right)_{\Omega_{2}} \quad \forall \mu \in \Lambda .
\end{aligned}
$$

For given $\lambda \in \Lambda$ we now consider for $i=1,2$ the harmonic extensions $u_{i}^{0}=H_{i}\left(\kappa_{i}(\lambda)\right) \in V_{i}$ of the Dirichlet boundary value $\kappa_{i}(\lambda)$ on $\Gamma$. (From now on, the brackets are mostly skipped to simplify the notation.) Furthermore let $u_{i}^{*}=\mathcal{G}_{i} f$ be the solutions of the subproblems (9) with homogeneous Dirichlet data $u_{i \mid \partial \Omega}^{0}=0$. Due to the linearity of the local problems (9) the functions $u_{i}=H_{i} \kappa_{i} \lambda+\mathcal{G}_{i} f$ satisfy (9)-(11) if and only if

$$
\begin{aligned}
& a_{1}\left(H_{1} \kappa_{1} \lambda, R_{1} \mu\right)+a_{2}\left(H_{2} \kappa_{2} \lambda, R_{2} \mu\right)= \\
& \quad\left(f, R_{1} \mu\right)_{\Omega_{1}}-a_{1}\left(\mathcal{G}_{1} f, R_{1} \mu\right)+\left(f, R_{2} \mu\right)_{\Omega_{2}}-a_{2}\left(\mathcal{G}_{2} f, R_{2} \mu\right) \quad \forall \mu \in \Lambda .
\end{aligned}
$$

Since the extension operators $R_{i}, i=1,2$, can be chosen arbitrarily, we set $R_{i}=H_{i}$. Denoting by $\langle\cdot, \cdot\rangle$ the duality pairing between $\Lambda^{\prime}$ and $\Lambda$ we recall the definition of the Steklov-Poincaré operators $S_{i}: \Lambda \rightarrow \Lambda^{\prime}$ :

$$
\left\langle S_{i} \eta, \mu\right\rangle=a_{i}\left(H_{i} \eta, H_{i} \mu\right) \quad \forall \eta, \mu \in \Lambda, \quad i=1,2
$$

and furthermore the functional $\chi=\chi_{1}+\chi_{2} \in \Lambda^{\prime}$ :

$$
\left\langle\chi_{i}, \mu\right\rangle=\left(f, H_{i} \mu\right)_{\Omega_{i}}-a_{i}\left(\mathcal{G}_{i} f, H_{i} \mu\right) \quad \forall \mu \in \Lambda, \quad i=1,2 .
$$

Now (12) can be written as the nonlinear Steklov-Poincaré interface equation

$$
\text { find } \lambda \in \Lambda: \quad\left(S_{1} \kappa_{1}+S_{2} \kappa_{2}\right) \lambda=\chi
$$


or equivalently

$$
\text { find } \lambda_{2} \in \Lambda: \quad\left(S_{1} \kappa_{1} \kappa_{2}^{-1}+S_{2}\right) \lambda_{2}=\chi
$$

if we set $\lambda_{2}=\kappa_{2} \lambda$. Note that if $\kappa_{2}^{-1}: \Lambda \rightarrow \Lambda$ is Lipschitz continuous the convergence of a sequence of iterates $\lambda_{2}^{k}$ to $\lambda_{2}$ implies the convergence of $\lambda^{k}=\kappa_{2}^{-1} \lambda_{2}^{k}$ to $\lambda$. We state the main result of this section:

Proposition 2. Solving problem (4)-(6) is equivalent to solving the nonlinear Steklov-Poincaré equations (15) or (16).

We point out at this stage that the reformulation of the problem (4)-(6) by Kirchhoff transformation is not only a powerful tool for the analysis of the problem that will be subject of the following two sections but also for its numerical treatment due to the linearity of transformed subproblems. In more complicated cases like the time-discretized Richards equation Kirchhoff transformation allows a reformulation of the quasilinear subproblems as convex minimization problems which can be solved efficiently and robustly using monotone multigrid methods [4].

\section{Nonlinear Dirichlet-Neumann iteration}

Now we consider the Dirichlet-Neumann algorithm applied to our problem (4)-(6). However, since it turns out that for a rigorous analysis the damping has to be carried out in the transformed variables, we state it for the transformed version (9)-(11):

Given $\lambda_{2}^{0} \in \Lambda$, successively find $u_{1}^{k+1} \in V_{1}$ and $u_{2}^{k+1} \in V_{2}$ for each $k \geq 0$ such that

$$
\begin{aligned}
& a_{1}\left(u_{1}^{k+1}, v_{1}\right)=\left(f, v_{1}\right)_{\Omega_{1}} \quad \forall v_{1} \in V_{1}^{0} \\
& u_{1 \mid \Gamma}^{k+1}=\kappa_{1} \kappa_{2}^{-1}\left(\lambda_{2}^{k}\right) \quad \text { in } \Lambda \\
& a_{2}\left(u_{2}^{k+1}, v_{2}\right)=\left(f, v_{2}\right)_{\Omega_{2}} \quad \forall v_{2} \in V_{2}^{0} \\
& a_{2}\left(u_{2}^{k+1}, H_{2} \mu\right)-\left(f, H_{2} \mu\right)_{\Omega_{2}}=-a_{1}\left(u_{1}^{k+1}, H_{1} \mu\right)+\left(f, H_{1} \mu\right)_{\Omega_{1}} \quad \forall \mu \in \Lambda \text {. }
\end{aligned}
$$

Then, with some damping parameter $\theta \in(0,1)$, the new iterate is

$$
\lambda_{2}^{k+1}:=\theta u_{2 \mid \Gamma}^{k+1}+(1-\theta) \lambda_{2}^{k} .
$$

Considering the harmonic extensions $H_{i} u_{i \mid \Gamma}^{k+1}$ and the solutions $\mathcal{G}_{i} f$ of the problems (9) with homogeneous boundary data for $i=1,2$ the intermediate iterates are obtained by

$$
u_{1}^{k+1}=H_{1} \kappa_{1} \kappa_{2}^{-1} \lambda_{2}^{k}+\mathcal{G}_{1} f \quad \text { and } \quad u_{2}^{k+1}=H_{2} u_{2 \mid \Gamma}^{k+1}+\mathcal{G}_{2} f .
$$

Thus equation (20) provides 


$$
\begin{aligned}
& a_{1}\left(H_{1} \kappa_{1} \kappa_{2}^{-1} \lambda_{2}^{k}, H_{1} \mu\right)+a_{2}\left(H_{2} u_{2 \mid \Gamma}^{k+1}, H_{2} \mu\right) \\
& \quad=\sum_{i=1}^{2}\left(f, H_{i} \mu\right)_{\Omega_{i}}-a_{i}\left(\mathcal{G}_{i} f, H_{i} \mu\right) \quad \forall \mu \in \Lambda,
\end{aligned}
$$

which is the same as

$$
\left\langle S_{2} u_{2 \mid \Gamma}^{k+1}, \mu\right\rangle=\left\langle-S_{1} \kappa_{1} \kappa_{2}^{-1} \lambda_{2}^{k}+\chi, \mu\right\rangle \quad \forall \mu \in \Lambda
$$

and regarding (21) altogether yields

$$
S_{2}\left(\lambda_{2}^{k+1}-\lambda_{2}^{k}\right)=\theta\left(\chi-\left(S_{1} \kappa_{1} \kappa_{2}^{-1}+S_{2}\right) \lambda_{2}^{k}\right) \text { in } \Lambda .
$$

Consequently the damped Dirichlet-Neumann algorithm applied to (9)-(11) is a preconditioned Richardson procedure for the nonlinear Steklov-Poincaré formulation (16) with $S_{2}$ as a preconditioner.

Note that an analogous formulation for the interface equation (15) can't be obtained due to the nonlinearity of $S_{2} \kappa_{2}$. However, (25) can be treated just as in the linear case if we apply the following generalization of an abstract convergence result in [5, pp. 118/9]. Let $X$ be a Hilbert space, $Q_{1}: X \rightarrow X^{\prime}$ a not necessarily linear operator and let $Q_{2}: X \rightarrow X^{\prime}$ be linear and invertible. With the definition $Q:=Q_{1}+Q_{2}$ and for given $G \in X^{\prime}$ we consider the problem

$$
\text { find } \lambda \in X: \quad Q \lambda=G
$$

together with the corresponding Richardson iteration

$$
\lambda^{k+1}=\lambda^{k}+\theta\left(G-Q \lambda^{k}\right) .
$$

Theorem 1. Let $Q_{2}$ be continuous and coercive, i.e. there are positive constants $\beta_{2}$ and $\alpha_{2}$ such that

$$
\left\langle Q_{2} \eta, \mu\right\rangle \leq \beta_{2}\|\eta\|_{X}\|\mu\|_{X} \quad \forall \eta, \mu \in X, \quad\left\langle Q_{2} \eta, \eta\right\rangle \geq \alpha_{2}\|\eta\|_{X}^{2} \quad \forall \eta \in X .
$$

Let $Q_{1}$ be Lipschitz continuous, i.e. there is a constant $\beta_{1}>0$ such that

$$
\left\langle Q_{1} \eta-Q_{1} \mu, \lambda\right\rangle \leq \beta_{1}\|\eta-\mu\|_{X}\|\lambda\|_{X} \quad \forall \eta, \mu, \lambda \in X .
$$

Suppose there exists a constant $\kappa^{*}>0$ such that

$$
\left\langle Q_{2}(\eta-\mu), Q^{-2}(Q \eta-Q \mu)\right\rangle+\langle Q \eta-Q \mu, \eta-\mu\rangle \geq \kappa^{*}\|\eta-\mu\|_{X}^{2} \quad \forall \eta, \mu \in X .
$$

Then (26) has a unique solution $\lambda \in X$. Furthermore for any given $\lambda^{0} \in X$ and any $\theta \in\left(0, \theta_{\max }\right)$ with

$$
\theta_{\max }:=\frac{\kappa^{*} \alpha_{2}^{2}}{\beta_{2}\left(\beta_{1}+\beta_{2}\right)^{2}}
$$

the sequence given by (27) converges in $X$ to $\lambda$. 
The proof is an application of Banach's fixed point theorem and can be carried out along the lines of the one given in [5, pp. 118/9], see also [2].

Remark 1. Note that condition (30) reduces to a much simpler expression if $Q_{2}$ is symmetric. In the linear case (30) is just the coerciveness of $Q_{1}$. In our nonlinear case, (30) states a uniform monotonicity of $Q_{1}$ of the form

$$
\left\langle Q_{1} \eta-Q_{1} \mu, \eta-\mu\right\rangle \geq \frac{\kappa^{*}}{2}\|\eta-\mu\|_{X}^{2} \quad \forall \eta, \mu \in X .
$$

Now, it is well known that in the particular situation of (16) and (25) both Steklov-Poincaré operators $S_{1}$ and $S_{2}$ are symmetric, continuous and coercive. Thus in order to apply Theorem 1 to the case $X=\Lambda, G=\chi, Q_{2}=S_{2}$ and $Q_{1}=S_{1} \kappa_{1} \kappa_{2}^{-1}$ we have to make sure that the conditions (29) and (32) are satisfied for $Q_{1}=S_{1} \kappa_{1} \kappa_{2}^{-1}$. So we arrive at the following

Theorem 2. The nonlinear Steklov-Poincaré equation (16) has a unique solution $\lambda_{2}$ in $\Lambda$ to which the nonlinear Dirichlet-Neumann scheme (17)-(21) converges for sufficiently small $\theta$ and any $\lambda_{2}^{0} \in \Lambda$ if the following two conditions are satisfied:

$\kappa_{1} \kappa_{2}^{-1}: \Lambda \rightarrow \Lambda$ is Lipschitz continuous, i.e., there is a constant $L\left(\kappa_{1} \kappa_{2}^{-1}\right)>0$ such that

$$
\left\|\kappa_{1} \kappa_{2}^{-1} \eta-\kappa_{1} \kappa_{2}^{-1} \mu\right\|_{\Lambda} \leq L\left(\kappa_{1} \kappa_{2}^{-1}\right)\|\eta-\mu\|_{\Lambda} \quad \forall \eta, \mu \in \Lambda,
$$

and $S_{1} \kappa_{1} \kappa_{2}^{-1}: \Lambda \rightarrow \Lambda^{\prime}$ is a uniformly monotone operator, i.e. there is a constant $\alpha_{1}>0$ such that

$$
\left\langle S_{1}\left(\kappa_{1} \kappa_{2}^{-1} \eta-\kappa_{1} \kappa_{2}^{-1} \mu\right), \eta-\mu\right\rangle \geq \alpha_{1}\|\eta-\mu\|_{\Lambda}^{2} \quad \forall \eta, \mu \in \Lambda .
$$

Proposition 3. The conditions (33) and (34) are satisfied in one space dimension.

Proof. Let $\Omega_{1}=[a, b], \Omega_{2}=[b, c]$, with $\Gamma=\{b\}$ and $a<b<c$. Then we have $\Lambda=H_{00}^{1 / 2}(\Gamma)=H^{1 / 2}(\Gamma) \cong(\mathbb{R},|\cdot|)$ and condition (33) follows from Proposition 1 .

Let $L\left(\kappa_{1}^{-1}\right)$ and $L\left(\kappa_{2}\right)$ be the Lipschitz constants of the real functions $\kappa_{1}^{-1}$ and $\kappa_{2}$ according to Proposition 1 . In order to prove (34), let $\eta, \mu, \lambda \in \mathbb{R}$. The harmonic extension $H_{1}(\lambda)$ is the affine function $x \mapsto \frac{\lambda}{b-a} x-\frac{\lambda}{b-a} a$. As $\kappa_{1}^{-1}$ and $\kappa_{2}$ are monotonically increasing, we then have

$$
\begin{aligned}
\left\langleS _ { 1 } \left(\kappa_{1} \kappa_{2}^{-1} \eta-\kappa_{1}\right.\right. & \left.\left.\kappa_{2}^{-1} \mu\right), \eta-\mu\right\rangle \\
& =\int_{a}^{b} \nabla H_{1}\left(\kappa_{1} \kappa_{2}^{-1} \eta-\kappa_{1} \kappa_{2}^{-1} \mu\right) \nabla H_{1}(\eta-\mu) d x \\
& =\int_{a}^{b} \frac{\kappa_{1} \kappa_{2}^{-1} \eta-\kappa_{1} \kappa_{2}^{-1} \mu}{b-a} \cdot \frac{\eta-\mu}{b-a} d x \\
& =\frac{\left(\kappa_{1} \kappa_{2}^{-1} \eta-\kappa_{1} \kappa_{2}^{-1} \mu\right)(\eta-\mu)}{b-a} \\
& \geq \frac{1}{(b-a) L\left(\kappa_{1}^{-1}\right) L\left(\kappa_{2}\right)}|\eta-\mu|^{2}
\end{aligned}
$$


Remark 2. Unfortunately, in higher dimensions condition (34) is violated to the extend that $\left\langle S_{1}\left(\kappa_{1} \kappa_{2}^{-1} \eta-\kappa_{1} \kappa_{2}^{-1} \mu\right), \eta-\mu\right\rangle$ can be negative. A counterexample in $2 \mathrm{D}$ is easily obtained by considering a harmonic function $u$ with $\frac{\partial u}{\partial \mathbf{n}} \cdot u \leq c<0$ on a subset of $\Gamma$ with positive Hausdorff measure (see [2]).

\section{Numerical example}

In this section we apply our nonlinear Dirichlet-Neumann method to a problem in two space dimensions: We consider the transmission problem (1)-(3) on the Yin Yang domain $\Omega$ as shown in Figure 2. We denote the white subdomain together with the grey circle $B_{1}$ by $\Omega_{1}$ and the grey subdomain with the white circle $B_{2}$ by $\Omega_{2}$.

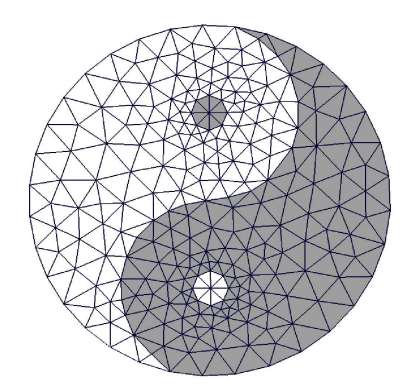

Fig. 2. Yin Yang domain $\Omega$.

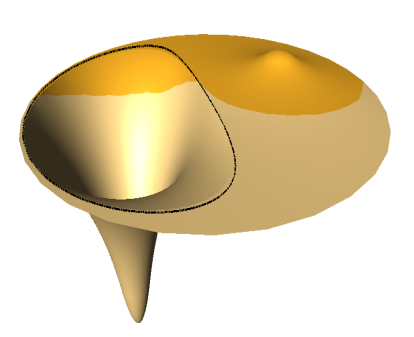

Fig. 3. Solution $p$ on $\Omega$ with free boundary (black line).

We select the data

$$
f(x)=(-1)^{i} \text { on } B_{i}, i=1,2, \quad f(x)=0 \text { elsewhere }
$$

and the nonlinearities

$$
k_{i}\left(p_{i}\right)=\left\{\begin{array}{cl}
K_{h} \max \left\{p_{i}^{3 \lambda_{i}+2}, c\right\} & \text { for } p_{i} \leq-1 \\
1 & \text { for } p_{i} \geq-1
\end{array}\right.
$$

This choice is motivated by the well-known state equations of Brooks-Corey and Burdine (cf. [6]) for the hydraulic conductivity of a saturated/unsaturated porous media with different soils. In this way, our model problem can be regarded as a stationary Richards equation. Note that $p_{i}<-1$ characterizes the unsaturated region which is separated by a free boundary from the linear, saturated regime occuring for $p_{i} \geq-1$. The parameters $\lambda_{1}$ and $\lambda_{2}$ in $\Omega_{1}$ resp. $\Omega_{2}$ are called pore size distribution factors. We chose them in an extreme manner as $\lambda_{1}=1.0$ (very coarse sand) and $\lambda_{2}=0.1$ (fine clay). The factor 
$K_{h}=0.002$ is a realistic hydraulic conductivity in the case of full saturation. The parameter $c=0.1>0$ is introduced to enforce ellipticity.

The choice of the data $f$ which result in a strong sink on $B_{1}$ and a strong source on $B_{2}$ and our special choice of $\bar{\Omega}_{1}$ and $\bar{\Omega}_{2}$ ensure that the free boundary has a nontrivial intersection with the interface $\Gamma=\bar{\Omega}_{1} \cap \bar{\Omega}_{2}$ (see the numerical solution as shown in Figure 3). Since we apply the Dirichlet-Neumann scheme (17)-(21) we hereby make sure that step (18) is nonlinear.

We discretize the problem on the two subdomains using piecewise linear finite element spaces. The linear problems on the subdomains are solved by a linear multigrid method. Figure 4 shows the convergence rate $\rho$ measured in

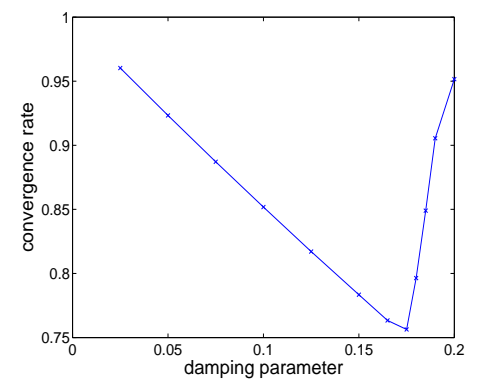

Fig. 4. $\rho$ vs. damping parameter $\theta$.

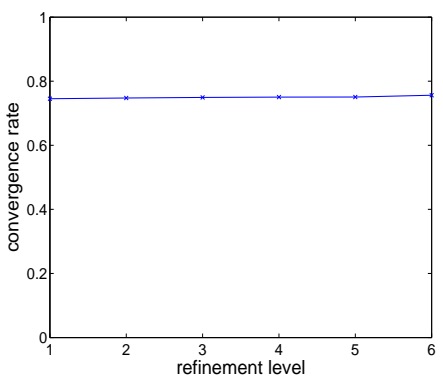

Fig. 5. $\rho$ vs. refinement level.

the energy norm (for the transformed variables $u_{i}^{k}$ ) with respect to the damping parameter $\theta$. We use a grid hierarchy of six levels as resulting from uniform mesh refinement of the coarse grid depicted in Figure 2. In this way, we obtain about 235.000 nodes on the finest mesh. Figure 5 shows the convergence rate over the refinement levels. The damping parameter $\theta_{\text {opt }}=0.175$ is obtained from Figure 4. For this optimal choice, we observe mesh-independence of the convergence speed.

\section{References}

1. H.W. Alt and S. Luckhaus. Quasilinear elliptic-parabolic differential equations. Math. Z., 183:311-341, 1983.

2. H. Berninger. Domain Decomposition Methods for Elliptic Problems with Jumping Nonlinearities. PhD thesis, Freie Universität Berlin, in preparation.

3. H. Berninger and M. Discacciati. Nonlinear Robin algorithms for quasilinear transmission problems. In preparation.

4. R. Kornhuber. On constrained Newton linearization and multigrid for variational inequalities. Numer. Math., 91:699-721, 2002.

5. A. Quarteroni and A. Valli. Domain Decomposition Methods for Partial Differential Equations. Oxford Science Publications, 1999.

6. M.T. van Genuchten. A closed-form equation for predicting the hydraulic conductivity of unsaturated soils. Soil Sci. Soc. Am. J., 44:892-898, 1980. 